\title{
Low-Dimensional Thermoelectricity
}

\author{
J.P. HEREMANS
}

Department of Mechanical Engineering and Department of Physics The Ohio State University, Columbus, Ohio 43202, USA

Thermoelectric materials are used as solid-state heat pumps and as power generators. The low efficiency of devices based on conventional bulk thermoelectric materials confines their applications to niches in which their advantages in compactness and controllability outweigh that drawback. Recent developments in nanotechnologies have led to the development of thermoelectric nano-materials with double the efficiency of the best bulk materials, opening several new classes of applications for thermoelectric energy conversion technology. We review here first the physical mechanisms that result in the superior thermoelectric performance of low-dimensional solids, compared to bulk thermoelectric materials: they are a reduction of the lattice thermal conductivity, and an increase in the Seebeck coefficient $S$ for a given carrier density. The second part of this review summarizes experimental results obtained on macroscopic arrays of bismuth, antimony, and zinc nanowires with diameters ranging from 200 to $7 \mathrm{~nm}$. We show how size-quantization effects greatly increase $S$ for a given carrier concentration, as long as the diameter of the nanowires remains above $9 \mathrm{~nm}$, below which localization effects start dominating. In a third part, we give data on $\mathrm{PbTe}$ nanocomposites, particularly bulk samples containing $30 \mathrm{~nm}$ diameter $\mathrm{Pb}$ inclusions. These inclusions affect the electron scattering in such a way as to again increase the Seebeck coefficient.

PACS numbers: 72.10.-d, 73.63.-b, 81.07.-b

\section{Introduction and motivation}

Thermoelectricity (TE) is one of the simplest technologies applicable to energy conversion. Thermoelectric materials can generate electrical power from heat, and use electricity to function as heat pumps providing active cooling or heating. The working fluid in these energy converters consists of the conduction electrons. The technology is entirely solid-state, and thermoelectric systems are simple compared to conventional mechanical systems; however, this simplicity comes at a price. Firstly, the complexity of the energy conversion problem is shifted to the solid-state physics aspects of the materials themselves. Secondly, the efficiency of conventional TE systems is far inferior to that of equivalent mechanical systems. The conventional wisdom is therefore that TE technology is limited to niche 
applications where its advantages of compactness, reliability and ease of control overcome its lack of efficiency. The recent developments in nanotechnologies that are reviewed in this paper hold the promise to change that situation, and to lead to the development of new large scale and environmentally-friendly applications in cooling technology and in power generation.

Thermoelectric energy conversion dates back to the period when Telkes [1], Ioffe $[2,3]$ and Goldsmid [4] applied the newly developed knowledge of transport in semiconductors to the problem. The technology developed at that time still dominates today [5], except for progress made in skutterudite materials [6]. It was recognized $[7,8]$ in the 1990's that low-dimensional systems should result in materials with much better efficiencies than bulk materials, through low-dimensional effects on both charge carriers and lattice waves. Such improvements have been experimentally demonstrated on $\mathrm{Bi}_{2} \mathrm{Te}_{3} / \mathrm{Sb}_{2} \mathrm{Te}_{3}$ superlattices [9] and on $\mathrm{PbTe} / \mathrm{PbSeTe}$ and $\mathrm{PbTe} / \mathrm{PbSnSeTe}$ quantum dot superlattices [10] in the early 2000's: the proof of principle that TE technology can indeed compete efficiency-wise with mechanical cooling systems and power generators thus exists. Research is needed to develop bulk material preparation techniques of nano-scale materials, such as self-assembly techniques, that will make this a commercial reality.

The basic material property governing the efficiency of thermoelectric energy converters is the thermoelectric figure of merit [4]:

$$
Z=\frac{S^{2} \sigma}{\kappa},
$$

where $S$ is the Seebeck coefficient, also called the thermoelectric power, $\sigma$ is the electrical conductivity and $\kappa$ is the thermal conductivity. $Z$ is usually multiplied by the average temperature $T$ to yield a dimensionless number $Z T$. Conventional thermoelectric materials, most of which were developed in the 1960's, have been limited to $Z T=1$ at $T=300 \mathrm{~K}$. The new nano-scale materials, in particular quantum-dot superlattices (QDSL), have reached $Z T=2$ at $300 \mathrm{~K}$ [10], and even $Z T=3$ at $450 \mathrm{~K}$ [11]. The results are significant: we shall now show that the value of $Z T=2$ is near the threshold [12] at which one can envision large-scale applications of thermoelectricity with performances comparable to those of conventional mechanical machines.

The equations relating $Z T$ to the efficiency of a TE system generating electrical power from a heat source are given in the classical monographs on thermoelectric applications [4]. In essence, the efficiency consists of the Carnot factor (determined by the temperature gradient across the generator), multiplied by a thermoelectric efficiency factor that is a function of $Z T$. An application for thermoelectric generators that is the subject of research today is the recovery of heat wasted by automobile engines. It is known that the chemical energy in an automotive fuel is distributed into three roughly equal parts: (1) mechanical power transmitted to the wheels, (2) heat dissipated in the radiator, and (3) heat exhausted through the tailpipe. Therefore, if the heat wasted in the exhaust could 
be converted into electrical power with a given efficiency $\eta$ (and put to good use for instance in auxiliary electrical propulsion), the efficiency of the vehicle would be increased by the same amount $\eta$. Let us consider, for example, the case of an automotive electric generator operating using the exhaust gases on a typical passenger car, downstream from the catalytic converter. The high-temperature end of the TE elements could reach $250^{\circ} \mathrm{C}$ (including the losses in the heat exchanger between the gases and the elements) and the cold side could be maintained at $100^{\circ} \mathrm{C}$ so as to avoid condensing water. One can calculate [4] that with $Z T=1$, $\eta=6 \%$, but with $Z T=2, \eta=10 \%$. Such an improvement in fuel economy would be very considerable.

The second large-scale application involved cooling and refrigeration. The "efficiency" of thermoelectric heat pumps is given by the coefficient of performance (COP). The COP is defined as the amount of heat pumped at the cold end of a TE refrigerator, or at the hot end of a TE heating element, divided by the amount of electrical power used. Once again, the equations relating the COP of the system to the $Z T$ of the material are given elsewhere [4] for single Peltier elements and classical TE modules. We consider, for example, the case of automotive climate control/air conditioning. The present vapor-compression mobile air-conditioning systems use a fluorocarbon (R-134a) as cooling fluid. It is expected that R-134a must be replaced because of its potential as a greenhouse gas. This creates an opportunity for TE cooling to replace R-134a cooling, if the efficiency, power, and economical requirements of the system can be met. Reference [12] shows a comparison of the maximum COP of a thermoelectric cooler with that of vapor-compression systems calculated for a temperature gradient of $40^{\circ} \mathrm{C}$, as a function of the thermoelectric figure of merit, $Z T$, of the material. Using classical TE modules, with $Z T=1$, the COP of a thermoelectric cooler is only $25 \%$ of that of a vapor-compression cooler, and thus this technology cannot be used. However, by combining recent ideas in "segmentation" of heat exchangers [13, 14], with new TE materials that have a $Z T=2$, the COP of TE coolers can reach that of vapor-compression coolers in climate-control applications. Once the efficiency issue is overcome, TE cooling has several additional advantages: (1) it is particularly favorable to rapid transient cooling [15]; (2) TE coolers are easy to control proportionally by varying the current, and therefore can be operated in either steady-state mode for maximum COP, or can be switched to operate in transient mode for maximum cooling power; (3) TE coolers are very compact and can be distributed across the vehicle, providing cold air directly to the occupants. Since a TE-cooling system cannot cost more than a conventional cooling system in order to be economically viable, these considerations set realistic quantitative goals to discover a synthesis technique that can produce large quantities of thermoelectric nano-scale material with $Z T=2$.

How do low-dimensional materials improve the $Z T$ ? In bulk materials, all three properties that govern $Z T, S, \sigma$, and $\kappa$ are related. For instance, by increas- 
ing the carrier density, $n$, one does increase the electrical conductivity, but this decreases the Seebeck coefficient. In fact, the numerator of Eq. (1), $S^{2} \sigma$, which is also referred to as the "power factor", is optimized [4] for degenerately-doped narrow-gap semiconductors with $n \approx 10^{19} \mathrm{~cm}^{-3}$, and a value of $S=170 \mu \mathrm{V} / \mathrm{K}$. The thermal conductivity $\kappa$ of such semiconductors is dominated by heat conduction through phonons, $\kappa_{\mathrm{L}}$. The mechanisms that decrease $\kappa_{\mathrm{L}}$ such as alloying, do decrease the electron (or hole) mobility $\mu$ as well, thus affecting $\sigma$. It is the hallmark of low-dimensional thermoelectric materials that the introduction of a new design parameter, the characteristic length scale $d$ of the material, eases the limitations that arise from this interrelation. The characteristic length scale $d$ can be the thickness of the quantum well in a two-dimensional system (2D), the diameter of a quantum wire in a $1 \mathrm{D}$ system, or of a quantum dot in a $0 \mathrm{D}$ system. There are three mechanisms through which this works.

Firstly, low-dimensional systems can have a reduced lattice thermal conductivity $\kappa_{\mathrm{L}}$ without too high a loss in mobility $\mu$. This may arise because the phonon mode structure is affected by the low-dimensionality, or because the phonon mean free path $\ell_{\varphi}$ is limited by $d$, while the electron mean free path $\ell_{\mathrm{e}}$ is less so. Obviously, this requires that the condition $\ell_{\varphi} \approx d \geq \ell_{\mathrm{e}}$ hold.

Secondly, the Seebeck coefficient in low-dimensional systems at a given carrier concentration is expected to be enhanced over that of $3 \mathrm{D}$ systems, for two reasons: size-quantization effects and electron energy filtering. To explain this, we refer to the Mott relation for the Seebeck coefficient. We can associate an electrical conductivity $\sigma(E)$ to the electrons that fill the energy levels between $E$ and $E+\mathrm{d} E$, irrespective of the mechanism that limits $\sigma(E)$. The total electrical conductivity is then the integral of this over the entire energy range, moderated by the Fermi distribution function $f_{0}(E)$. Integrating by parts, the total conductivity is

$$
\sigma=\int_{0}^{\infty} \sigma(E)\left(-\frac{\partial f_{0}(E)}{\partial E}\right) \mathrm{d} E .
$$

Cutler and Mott [16] derive the Seebeck coefficient in this formalism to be the differential form of the Mott relation

$$
S=\frac{k_{\mathrm{B}}}{q} \frac{1}{\sigma} \int_{0}^{\infty} \sigma(E)\left(\frac{E-E_{\mathrm{F}}}{k_{\mathrm{B}} T}\right)\left(\frac{\partial f_{0}(E)}{\partial E}\right) \mathrm{d} E .
$$

In systems in which the Fermi statistics are degenerate, such as metals and degenerately doped semiconductors, Eq. (3) simplifies to the better-known form of the Mott relation

$$
S=\frac{\pi^{2}}{3} \frac{k_{\mathrm{B}}}{q} k_{\mathrm{B}} T\left\{\frac{\mathrm{d}[\ln (\sigma(E))]}{\mathrm{d} E}\right\}_{E=E_{\mathrm{F}}},
$$

which is generally valid, whether conduction is through band states, localized states, hopping or other mechanisms. In the particular case of band conduction, 
the differential conductivity can be expressed by the product of the density of carriers $n(E)$, which is a function of the density of states $g(E)$, and their differential mobility $\mu(E)$ or relaxation time $\tau(E)$ :

$$
\sigma(E)=n(E) e \mu(E)=n(E) e^{2} \frac{\tau(E)}{m^{*}},
$$

where $e$ is the free electron charge, and $m^{*}$ the effective mass (in solids with non-parabolic bands, $m^{*}=m^{*}(E)$ ). From Eqs. (3) or (4), it is clear that any mechanism that enhances the energy-dependence of the conductivity $\mathrm{d} \sigma(E) / \mathrm{d} E$, will enhance the Seebeck coefficient $S$. There are two ways to enhance $\mathrm{d} \sigma(E) / \mathrm{d} E$, namely enhancing $\mathrm{d} n(E) / \mathrm{d} E$ or enhancing $\mathrm{d} \mu(E) / \mathrm{d} E$. We now review the two mechanisms separately.

Increasing $\mathrm{d} n(E) / \mathrm{d} E$ can be achieved by enhancing the dependence of the density of states on energy, $\mathrm{d} g(E) / \mathrm{d} E$. The size-quantization effect does this in low-dimensional structures. Indeed, we show in Fig. 1 the energy dependence of the density of states in 3,2,1 and 0D structures. The density of states function

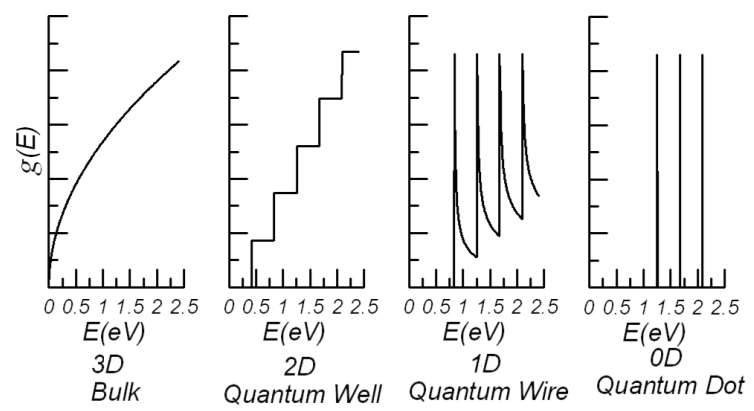

Fig. 1. Energy dependence of the electronic density of states in 3-, 2-, 1- and 0dimensional crystals.

results [17] from a dispersion relation $E(\boldsymbol{k})$ in which electrons have, as quantum numbers, a momentum $\boldsymbol{k}$ along 3, 2 or 1 axes of the crystal in 3, 2, or 1D, and a set of fixed quantum numbers $(1,2,3 \ldots)$ along directions in which the motion is constrained (1 direction in 2D systems, 2 directions in $1 \mathrm{D}$ systems, all 3 directions in $0 \mathrm{D}$ systems). It is quite obvious that the sharp maxima in $g(E)$ of quantum wires seen in Fig. 1 will result in a strong increase in the Seebeck coefficient via Eqs. (3) or (4). While the concept of thermoelectric transport in low-dimensions is quite old [18], the prediction that the figure of merit should be enhanced through this effect was behind the seminal papers by Hicks and Dresselhaus [7, 8]. Quantitative calculations of this effect, and its experimental verification, are given further for bismuth nanowires.

An increase in the energy-dependence of the mobility $\mathrm{d} \mu(E) / \mathrm{d} E$ can result from increasing the scattering time's energy dependence, $\mathrm{d} \tau(E) / \mathrm{d} E$, by preferentially scattering electrons depending on their energy: this is what we mean with 
electron energy filtering. In conventional semiconductors with parabolic bands, it is customary to ascribe a power-law dependence to the relaxation time

$$
\tau=\tau_{0} E^{\lambda-\frac{1}{2}} .
$$

The origin of this very coarse formula is as follows. The relaxation time is the inverse of the scattering probability, which is in turn determined by two factors: (1) the density of initial and final states, which gives rise in 3D solids to the factor $E^{1 / 2}$ (see Fig. 1), and (2) the probability matrix element which, very schematically, gives rise to the factor $E^{\lambda} . \lambda$ is the scattering exponent, approximated [19] for various scattering mechanisms as: $\lambda=0$ for scattering of electrons on acoustic phonons, $\lambda=1 / 2$ for scattering of electrons on neutral impurities, and $\lambda=2$ for scattering of electrons on ionized impurities. It was recognized since the early days of the development of semiconductor thermoelectric technology that an increase in $\lambda$ could be beneficial, and obviously ionized impurity scattering would effect that. Ioffe himself proposed [4] counter-doping PbTe to increase $\lambda$; unfortunately the mobility was much decreased [20] in counter-doped PbTe. In contrast to the PbTe case, the technique did appreciably enhance the $Z T$ of counter-doped bismuth [21].

Several other descriptions exist detailing how nanostructures can increase the Seebeck coefficient by filtering electrons according to their energy, using several other models besides scattering. We mention in particular the use of potential energy barriers in 2D structures [22], which can create minibands in the energy band structure. Such minibands could also filter electrons according to their energy. Another possibility is akin to hot electron injections from a potential barrier [23], which again selects carriers from a given energy range. When conceiving of semiconductor heterostructures for thermoelectricity, it is useful to bear in mind that only majority-carrier systems are advantageous, as any recombination mechanism with minority carriers is a loss [24] that detracts from the overall device efficiency.

The following two paragraphs summarize recent development in two low-dimensional thermoelectric systems, bismuth nanowires, and lead-telluride composites that include quantum dots.

\section{A quantum-wire system: bismuth nanowires}

\subsection{Theory}

In bulk, bismuth is a semimetal with a Brillouin zone and Fermi surfaces shown in Fig. 2a. In essence, the holes fill two half-pockets at the $T$-points of the Brillouin zone, and the electrons fill six half-pockets at the $L$-points; the energy level of the bottom of the conduction bands overlaps that of the top of the valence bands by $38 \mathrm{meV}$ at $4.2 \mathrm{~K}$. Lin et al. [25] consider the case of quantum wires of diameter $d$ prepared from $\mathrm{Bi}$, and with a long axis oriented along the crystallographic [0112] axis. This lifts the degeneracy between the electron pockets: one (or two halves) pocket presents its smallest cross-section to that orientation, and is labeled $L(A)$, the other two (four halves) present a larger cross-section and are labeled $L(B, C)$. The energy dependence of the band minima of these pockets, 

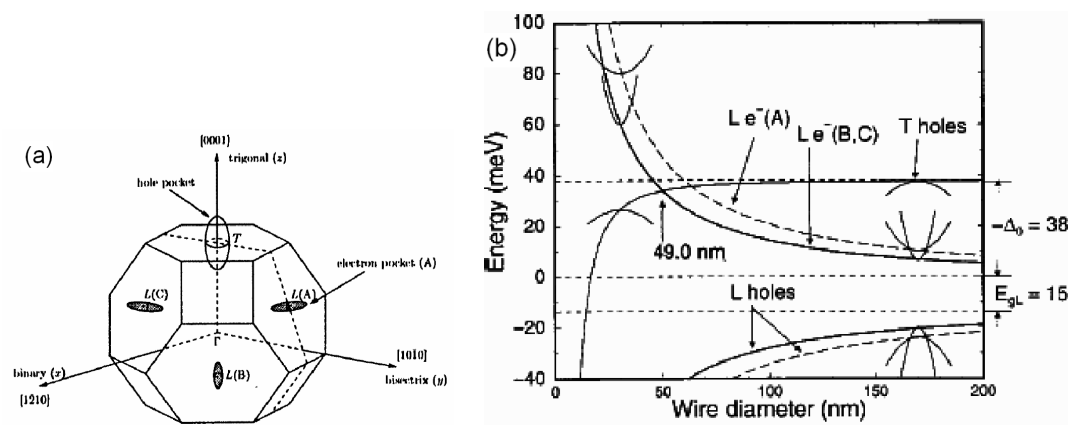

Fig. 2. Part (a) shows the Brillouin zone of bulk Bi, with the Fermi surfaces (labeled $A, B, C)$ of the electrons at the $L$-points and the holes at the $T$-points. A series of maxima in the band structure also exist at the $L$-points, so that $L$-point holes can exist in $p$-type doped samples or in Bi quantum wires. The [0112] direction is close to the one obtained by connecting the $\Gamma$ point with the $L(A)$ point in (a). Part (b) shows the subband structure at $77 \mathrm{~K}$ of Bi quantum wires oriented along the [0112] direction, as a function of wire diameter. It shows the energies of the highest subbands for the $T$-point hole carrier pocket as well as for the $L$-point holes, and the lowest subbands for the $L$-point electron pockets $(A, B$ and $C$ ). The zero energy refers to the conduction band edge in bulk Bi. The conduction subbands move up in energy as the wire diameter decreases, while the valence subbands move down. A semimetal-semiconductor transition occurs at a diameter of $49 \mathrm{~nm}$, where the highest $T$-point valence subband edge crosses the lowest conduction subband edge formed by the $L(B, C)$ electrons (figure reprinted with permission from Y.-M. Lin, X. Sun, M.S. Dresselhaus, Phys. Rev. B 62, 4610 (2000). Copyright (2000) by the American Physical Society).

and of the band maxima of the hole pockets, is shown [25] as a function of wire diameter in Fig. 2b. The electron and hole bands cross at a diameter near $49 \mathrm{~nm}$ : there is a metal to semiconductor transition in quantum wires at that diameter. Lin et al. [25] further calculate the thermoelectric figure of merit in $1 \mathrm{D}, Z T_{1 \mathrm{D}}$, for these quantum wires as a function of the position of the Fermi level. The results at $77 \mathrm{~K}$ are shown in Fig. 3 as a function of the density of electrons in $n$-type doped material. Two conclusions can be drawn. Firstly, as expected from the arguments in the introduction, the $Z T$ value is greatly enhanced over that of bulk Bi along the trigonal axis, for which $Z T=0.15$ at $100 \mathrm{~K}$ [26]. Secondly, $Z T$ is a very strong function of the exact doping level. This also can be understood intuitively: the enhancement of the Seebeck coefficient results from the sharp dependence of $g(E)$ upon $E$, but this effect is maximal when the Fermi energy aligns fairly exactly with the maxima in density of states shown for 1D systems in Fig. 1. As a result, the doping optimization is much more critical to the design of quantum-wire thermoelectric materials than it is to bulk TE materials. We now turn to a review of the experimental data on this system. 


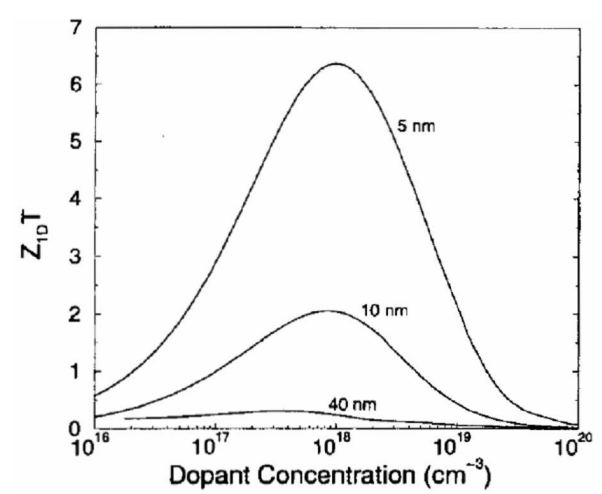

Fig. 3. Calculated one-dimensional figure of merit $Z_{1 \mathrm{D}} T$ for $n$-type doped bismuth nanowires, as a function of the electron density. The calculations are made at $77 \mathrm{~K}$, for wires oriented along the trigonal direction, for the three values of the nanowire diameter indicated (figure reprinted with permission from Y.-M. Lin, X. Sun, M.S. Dresselhaus, Phys. Rev. B 62, 4610 (2000). Copyright (2000) by the American Physical Society).

\subsection{Sample preparation}

All the data presented further are taken on macroscopic samples consisting of nanowires imbedded in insulating porous host materials. The host materials used are: (1) anodic porous alumina films grown electrochemically on metallic aluminum with oriented pores of diameters ranging from 7 to $200 \mathrm{~nm}$, (2) porous alumina grains with pores on the order of $9 \mathrm{~nm},(3)$ porous silica with pores of $15 \mathrm{~nm}, 8 \mathrm{~nm}$, and $4 \mathrm{~nm}$ (the latter is Vycor glass, $98 \% \mathrm{SiO}_{2}$ ). The anodic alumina (1) films contain regular arrays of aligned pores, all parallel to one another and oriented along the electrochemical growth axis. We review data on nanowires made from three host species, bismuth, and also antimony and zinc.

In the past, the most frequently used techniques to introduce nanometer diameter wires into porous host materials have used a high pressure to force molten metal into the pores [27] or used glass capillaries filled with molten $\mathrm{Bi}$, which were then extruded into narrow wires [28]. More recently, we described a vapor-phase method $[29,30]$ shown here in Fig. 4. The sample preparation process is as follows: in a cryopumped vacuum chamber, the porous host material is placed on top of a crucible which is resistively heated. A cover plate with a separate heater caps the assembly, which is held in place by a clip. In a first step, after the assembly has been pumped down to a base pressure of about $10^{-8}$ Torr, both heaters are used to outgas the host material at about $650^{\circ} \mathrm{C}$. In the second step, both heaters are set to an operating temperature of about $480^{\circ} \mathrm{C}$ for $\mathrm{Zn}$, and $590^{\circ} \mathrm{C}$ for both $\mathrm{Bi}$ and $\mathrm{Sb}$. The charge of the guest metal is heated so that metal vapor fills the crucible, passes through the porous host and escapes into the vacuum. After a few minutes, the temperature of the top heater is decreased first, and then the whole assembly is slowly cooled, while a temperature gradient across the porous host is 


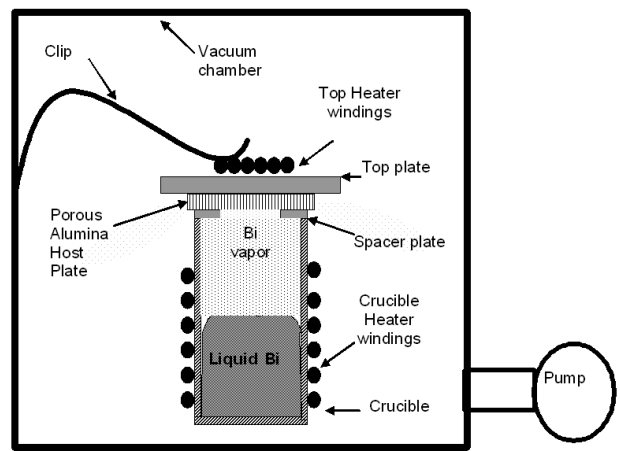

Fig. 4. Schematic diagram of the vapor-phase method to prepare nanowires in porous host materials.

maintained so that nanowires of the guest metal condense in the pores, from top to bottom. The process is stopped once the pores are filled with metal.

Samples prepared as described above are macroscopic nanocomposites, and can be handled and measured as bulk samples. Scanning electron micrographs of Bi-impregnated anodic $\mathrm{Al}_{2} \mathrm{O}_{3}(200 \mathrm{~nm})$ and $\mathrm{SiO}_{2}(15 \mathrm{~nm})$ are shown in Fig. 5 . The anodic alumina is a plate, $50 \mu \mathrm{m}$ thick, in which the pores are aligned and traverse the sample from end to end, as can be seen in Fig. 5a. The other host materials consist of many grains with pores randomly oriented. Two $\mathrm{SiO}_{2}$ grains are visible in Fig. 5b, with the pores visible as dark spots. Some pores are filled with $\mathrm{Bi}$, and a few $\mathrm{Bi}$ nanowires are seen extending out of the foreground grain in Fig. 5b. The samples can be mounted in conventional cryostats, and galvanomagnetic and thermoelectric transport coefficients are measured using classical steady-state methods.
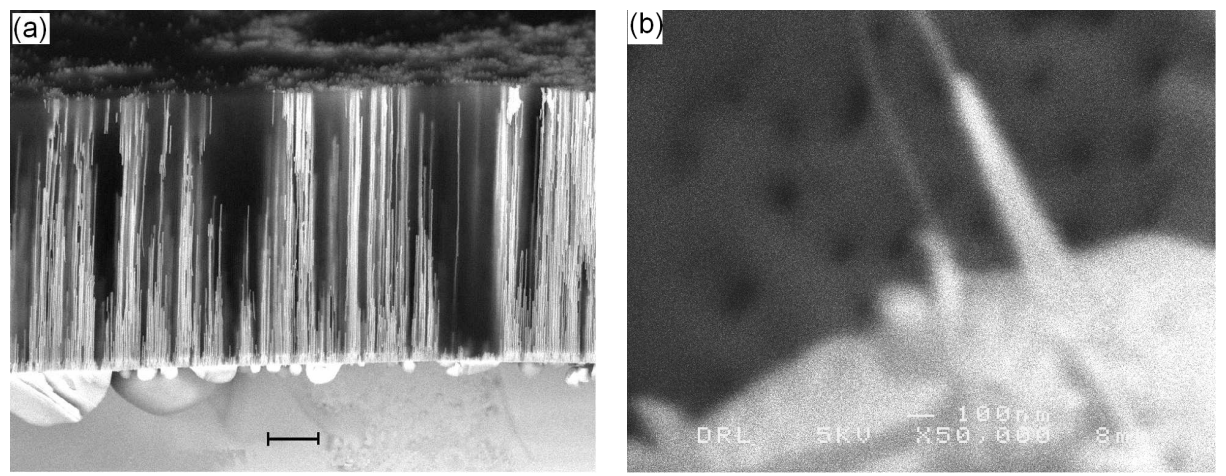

Fig. 5. Scanning electron micrographs of (a) aligned $\mathrm{Bi}$ nanowires in anodic $\mathrm{Al}_{2} \mathrm{O}_{3}$ with $d_{w}=200 \mathrm{~nm}$ and (b) $\mathrm{SiO}_{2}$ with $d_{w}=15 \mathrm{~nm}$ pores containing a random network of Bi nanowires, several of which can be seen protruding out of a grain. The scale marker in (a) is $10 \mu \mathrm{m}$, in (b) $100 \mathrm{~nm}$. 


\subsection{Electrical resistance versus temperature}

As it is impossible to assess how many nanowires are electrically connected through the nanocomposite, it is not possible to estimate the effective cross-section of the nanowires, and thus the electrical resistivity. Therefore, we show only the temperature dependence of the resistance of the nanocomposites, normalized to their room temperature $(300 \mathrm{~K})$ resistances. Figure 6 shows the temperature dependence of the resistance of nanowires in the various host materials, constructed from the data in several research papers. The data from aligned anodic $\mathrm{Al}_{2} \mathrm{O}_{3}$ composites with $d \geq 38 \mathrm{~nm}$ are from Ref. [29], those in composites with $d \leq 15 \mathrm{~nm}$ from [31] for the various diameters indicated. Two distinct behaviors are observed.

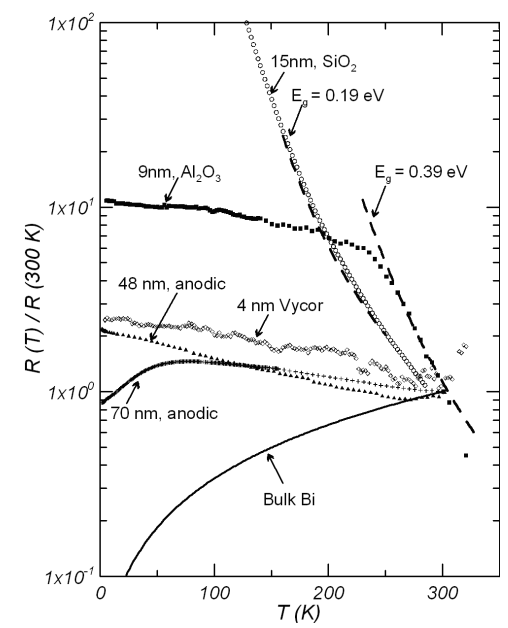

Fig. 6. Temperature dependence of aligned Bi nanowires in various host material, normalized to their value at $300 \mathrm{~K}$, for the diameters indicated. The resistance of bulk Bi is also shown. The data for wires of diameters are taken on samples in anodic $\mathrm{Al}_{2} \mathrm{O}_{3}$, Ref. [29]; the data on wires of diameters $15 \mathrm{~nm}$ are on composites based on porous $\mathrm{SiO}_{2}$ or $\mathrm{Al}_{2} \mathrm{O}_{3}$ with random pores (after Ref. [31]). The data are points; the lines are fits to Eq. (7).

Bulk Bi has a positive $\mathrm{d} R / \mathrm{d} T$ slope, because, even though the electron and hole densities increase by about one order of magnitude between 70 and $300 \mathrm{~K}$ in that semimetal [32], the phonon-limited mobility decreases with temperature almost as a $T^{-4}$ law [32]. The $200 \mathrm{~nm}$ and $70 \mathrm{~nm}$ diameter wires are also semimetals: they have $\mathrm{d} R / \mathrm{d} T>0$ below $100 \mathrm{~K}$, where the carrier density is rather temperature-independent and phonon scattering contributes to a negative temperature coefficient of mobility, even though scattering on the wire boundaries dominates. Above $100 \mathrm{~K}$, the carrier density increases with temperature in semimetallic Bi [32], and, since boundary scattering dominates, the temperature dependence of the mobility is much weaker than in bulk. Nanowires with diameters of $49 \mathrm{~nm}$ or less have 
a $\mathrm{d} R / \mathrm{d} T<0$ over the entire temperature range, implying that the carrier density is now temperature-dependent at all temperatures. This is evidence for the metal-to-semiconductor transition that occurs at a diameter of about $49 \mathrm{~nm}$ [25].

The $d=15 \mathrm{~nm}$ sample, and the $d=9 \mathrm{~nm}$ sample at high temperature, display an activated behavior, with an energy $E_{\mathrm{g}}$, following a law:

$$
R(T)=R_{0} \mathrm{e}^{-E_{\mathrm{g}} / k_{\mathrm{B}} T} .
$$

Fits of Eq. (7) to the data, along with the resulting values of $E_{\mathrm{g}}$, are given in Fig. 7. In this regime of temperatures and diameters, we believe that the behavior is that of a classical near-intrinsic semiconductor with band conduction and an energy gap $E_{\mathrm{g}}$.

The sample with $d=4 \mathrm{~nm}$, and the $d=9 \mathrm{~nm}$ sample at low temperature, display a temperature dependence that follows a $R(T) \propto T^{-1 / 2}$ power-law; we will show further that this is indicative of $1 \mathrm{D}$ localization.

\subsection{Optical reflectivity and diameter-dependence of the energy gap}

The optical reflectivity spectrum of a $\mathrm{Bi} / \mathrm{SiO}_{2}$ composite, with $8 \mathrm{~nm}$ pores was also reported [33] and shows the presence of an energy gap around $0.4 \mathrm{eV}$. We can now summarize in Fig. 7 the values obtained for $E_{\mathrm{g}}$ from both transport and optical measurements, for wires of different diameters. These experimental values are compared in Fig. 7 with the theoretical energy gap between the $L$-point conduction bands and the $T$-point valence band for semiconducting bismuth nanowires. This gap has been calculated in Fig. 2 of Ref. [25] for nanowires oriented along the $[01 \overline{1} 2]$ direction of $\mathrm{Bi}$, and can be calculated for wires along the trigonal direction as well (see Fig. 7). As the experimental data come from polycrystalline samples, we expect them to lie in between the two curves, as indeed is the case. In conclusion, the bismuth nanowires do show the metal-to-

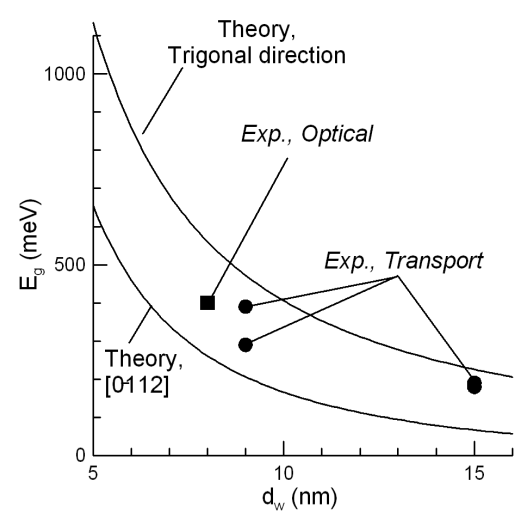

Fig. 7. Comparison of the experimental values of $E_{\mathrm{g}}$ obtained from Fig. 6 and Ref. [33] for various nanowire diameters, with the calculations of Ref. [25]. 
-semiconductor transition as predicted and the dependence of the energy gap on nanowire diameter in the semiconductor regime is also quite consistent with the calculations.

\subsection{Thermoelectric power}

The Seebeck coefficient of aligned metallic bismuth nanowires, $200 \mathrm{~nm}$ in diameter, imbedded in anodic alumina, has been reported [34] on several samples, including some of $n$-type doped bismuth. One curve, on intrinsic semimetallic $\mathrm{Bi}$, is reproduced here in Fig. 8. The Seebeck coefficient is essentially linear with

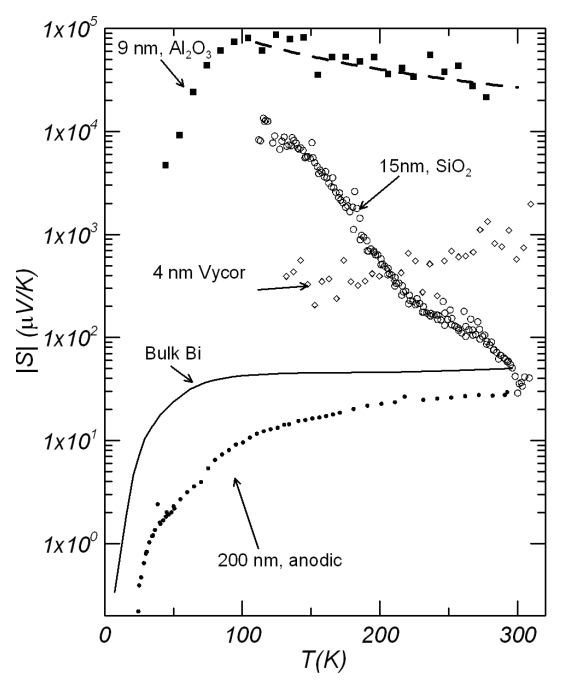

Fig. 8. Absolute value of the Seebeck coefficient of semimetallic bulk Bi [26] and $200 \mathrm{~nm}$

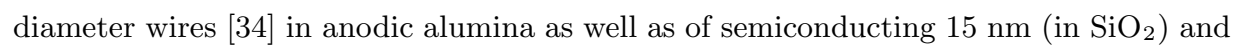
$9 \mathrm{~nm}$ (in $\mathrm{Al}_{2} \mathrm{O}_{3}$ ) diameter $\mathrm{Bi}$ nanowires, and finally of $4 \mathrm{~nm}$ diameter wires in Vycor glass [31]. The dashed line through the data on $9 \mathrm{~nm}$ Bi shows a $T^{-1}$ law.

temperature below about $90 \mathrm{~K}$, then flattens, as it does in bulk Bi [26] (also shown). Two samples of pure Bi nanowires, and one of $\mathrm{Bi} n$-type doped with Te, have been measured. In the original publication [34], the Shubnikov-de Haas measurements were taken on the same samples of these semimetallic wires, and their frequencies yielded the densities of electrons and holes in the samples. From these, assuming that the band structure of bulk Bi holds for wires of this large diameter, the authors calculated the partial electron and hole diffusion thermopowers, and, assuming that the partial mobilities scale as the effective masses, the total thermopower. Below $90 \mathrm{~K}$, the calculated results agree with the data remarkably well, given that there are no adjustable parameters in the calculation; the band structure of bismuth is known [35] to vary strongly with temperature above $100 \mathrm{~K}$.

The thermopower of semiconducting bismuth nanowires with diameters [31] from 15 to $9 \mathrm{~nm}$ is also reproduced here in Fig. 8, along with an extension to $4 \mathrm{~nm}$ 
diameter wires. A striking increase in the thermopower is observed as the wire diameter is decreased from $200 \mathrm{~nm}$ to $9 \mathrm{~nm}$, followed by a decrease for $4 \mathrm{~nm}$ wires. The temperature dependence of the $9 \mathrm{~nm}$ wires follows a $T^{-1}$ law at high temperatures, as expected for nearly intrinsic semiconductors, but the thermopower decreases at low temperatures. This behavior is consistent with the decreased thermopower observed in the $4 \mathrm{~nm}$ wires. The strong enhancement of the thermopower is only observed when the resistivity follows an activated behavior, Eq. (7), characteristic of a semiconductor, and not when the resistivity follows a $T^{-1 / 2}$ power law. We now ascribe this observation to localization effects.

\subsection{Localization effects}

Localization effects are most typically evidenced in magnetoresistance data, such as we show in Fig. 9 for $\mathrm{Bi}$ and $\mathrm{Zn}$ nanowires. Localization effects are quantum-mechanical corrections to the propagation of electrons. Considering only classical mechanics, any imperfection in a narrow quantum wire could completely block the passage of electrons at low temperature, and thus stop the electrical conductivity. In quantum mechanics, imperfections in a narrow wire can interfere with the wave functions of electrons propagating along that wire. These wave functions can then reconstruct constructively or destructively downstream from this imperfection. This results in an increase or decrease in the transmission probability of the electron across the imperfection. The determining factor for this effect is the length over which the electron wave functions conserve information about their phase, the phase coherence length $L_{\phi}$. The phase of the wave function is further affected by the amount of magnetic flux in the obstacle that interfered with the wave function, and therefore the magnetic flux affects the conductivity of the wire and generates magnetoresistance. These basic ideas are expressed in the following equation, which describes the variations of the electrical resistance $R_{0}$ in a one-dimensional system with magnetic field and temperature [36]:

$$
\begin{aligned}
& \frac{\Delta R(T, H)}{R_{0}}=\frac{e^{2} \rho_{\mathrm{e}}}{2 \pi \hbar A}\left[\frac{3}{2}\left(L_{\phi}^{-2}+\frac{4}{3} L_{\text {s.o. }}^{-2}+L_{H}^{-2}\right)^{-1 / 2}\right. \\
& \left.-\frac{1}{2}\left(L_{\phi}^{-2}+L_{H}^{-2}\right)^{-1 / 2}\right]
\end{aligned}
$$

as a function of the resistivity $\rho_{\mathrm{e}}$ of the wire, its cross-sectional area $A$, and the following lengths. $L_{H}$ is the magnetic length $(H=$ magnetic field):

$$
L_{H}=\sqrt{\frac{\hbar}{2 e H}} .
$$

$L_{\text {s.o. }}$ is the spin-orbit scattering length, important in Bi but much less so in lighter elements, such as Zn. $L_{\phi}$ is the phase-coherence length [36], the decay length that characterizes the loss of phase coherence of the electron wave function as it propagates further around the imperfection. It is responsible for the temperature dependence of the resistance and magnetoresistance, typically as a power law 

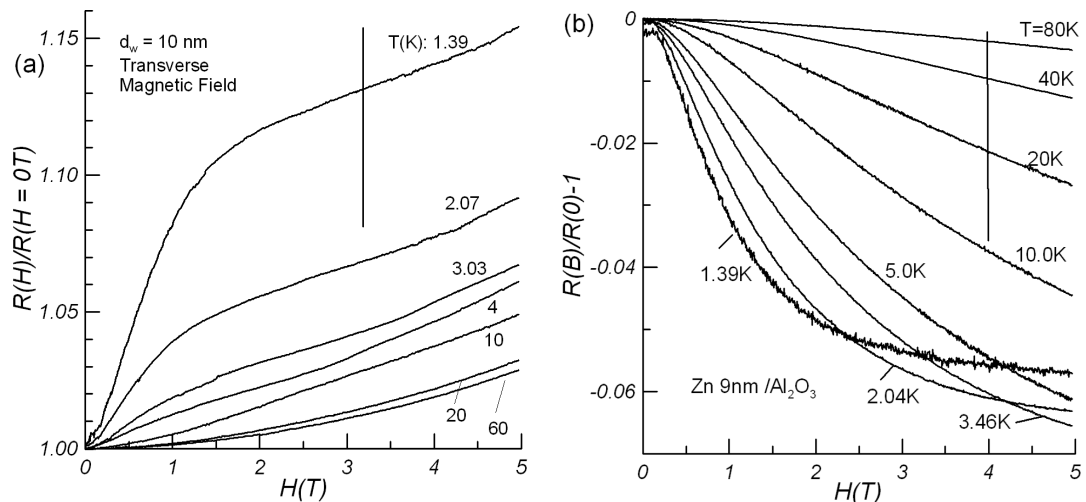

Fig. 9. Magnetic field dependence, at several temperatures, of the magnetoresistance of $10 \mathrm{~nm}$ diameter $\mathrm{Bi}$ (a) and $9 \mathrm{~nm}$ diameter $\mathrm{Zn}$ (b) nanowires. The Bi are oriented inside anodic alumina with the magnetic field oriented perpendicularly to the current direction. Very similar results are obtained in a parallel magnetic field. The $\mathrm{Zn}$ nanowires are inside randomly oriented pores in $\mathrm{Al}_{2} \mathrm{O}_{3}$. The vertical lines indicate the value of the magnetic field at which the flux inside a wire cross-section is one fluxon. The sign of the localization magnetoresistance is negative in $\mathrm{Zn}$, where spin-orbit interactions are weak, and positive in Bi, where they are strong (after Refs. [37, 33] and [39]).

$L_{\phi} \sim T^{-p} . L_{\phi}$ is limited by inelastic scattering events, for instance scattering by phonons for which $p=3 / 2$, or scattering by other electrons for which $p=1$.

The "step", or change in the slope, in magnetoresistance occurs [37] at a value of the magnetic field where the magnetic length $L_{H}$ equals the wire diameter, that is when one quantum of flux exactly fits inside a nanowire. This suggests that the step is due to one-dimensional localization (Eq. (8)). To further bolster the argument, we point out that the magnetic length, Eq. (9), depends only on the value of $H$ and not on any material parameter, such as the effective mass. Thus, the field at which these localization effects is observed should be independent of the solid from which the nanowires are made. The experiment was therefore repeated using $\mathrm{Sb}$ [38] and $\mathrm{Zn}$ [39] nanowires prepared in the same way as the Bi nanowires, and the magnetoresistance for $\mathrm{Bi}$ and $\mathrm{Zn}$ nanowires of similar diameter is shown in Fig. 9. The magnetoresistance step is consistently observed at the predicted magnetic field, irrespective of material. The data in Fig. 9 fit Eq. (8) very well [39]. The localization magnetoresistance in Zn, where spin-orbit interactions are negligible, is negative; in $\mathrm{Bi}$, where spin-orbit interactions dominate, it is positive. This effect is sometimes labeled "antilocalization".

The question is of course what becomes of the Seebeck coefficient in the localization regime. In order to answer that, it is advisable to dissociate the localization effects from the metal-to-semiconductor transition observed in $\mathrm{Bi}$, and this is the reason why the study was carried out on Zn nanowires, which are always metallic. The results are shown in Fig. 10, where we report the temperature 

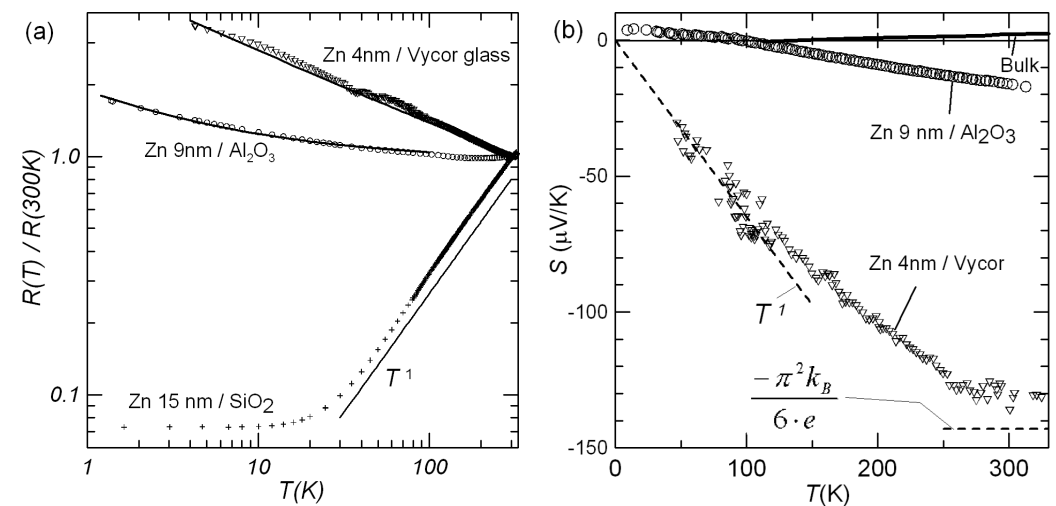

Fig. 10. Temperature dependence of (a) the normalized resistance and (b) of the Seebeck coefficient of Zn nanowires of the diameters indicated (after [39]).

dependence of the resistance (normalized to $300 \mathrm{~K}$ ) and of the thermopower of $\mathrm{Zn}$ nanowires of different diameters. The $15 \mathrm{~nm} \mathrm{Zn}$ wires are metallic, as evidenced by their positive $\mathrm{d} R / \mathrm{d} T$, and also by their magnetoresistance. The narrower wires have a resistance that follows a $T^{-1 / 2}$ law, as did the 4 and $9 \mathrm{~nm}$ diameter Bi nanowires; the magnetoresistance on those wires is, as we saw above, indicative of localized behavior. If we take this temperature dependence to be indicative of an energy dependence of the electrical conductivity as a power law $\sigma \propto E^{1 / 2}$, or, more precisely $\sigma \propto\left(E-E_{\mathrm{c}}\right)^{1 / 2}$, where $E_{\mathrm{c}}=k_{\mathrm{B}} T_{\mathrm{c}}$ is some critical energy, then, via the Mott equation (4), the Seebeck coefficient is

$$
\begin{aligned}
& S=-\pi^{2} k_{\mathrm{B}} / 6 q \quad \text { for } T>T_{\mathrm{c}}, \\
& S=-\pi^{2} k_{\mathrm{B}} T / 6 q T_{\mathrm{c}} \quad \text { for } T<T_{\mathrm{c}} .
\end{aligned}
$$

This prediction, shown in Fig. 10, again contains no adjustable parameters. It is very consistent with the data for the $4 \mathrm{~nm} \mathrm{Zn}$ nanowires, indicating again the validity of the Mott formula (4). It also shows that it is possible to achieve a thermopower on the order of $130 \mu \mathrm{V} / \mathrm{K}$ in metallic nanowires.

In contrast to the case of $\mathrm{Zn}$ nanowires, the localization effects seen in the magnetoresistance (Fig. 9) of the $10 \mathrm{~nm}$ Bi nanowires is on the order of $15 \%$, only about a factor of 2 smaller than the change in resistance with temperature seen in Fig. 7 between $230 \mathrm{~K}$ and $1 \mathrm{~K}$ (the $T^{-1 / 2}$ part of $R(T)$ ). Localization is expected to be more dominant yet in the $4 \mathrm{~nm} \mathrm{Bi} \mathrm{wires,} \mathrm{in} \mathrm{which} \mathrm{the} \mathrm{thermopower}$ is decreased in comparison to that of the $9 \mathrm{~nm}$ wires. We can conclude that the decrease in Seebeck coefficient seen in $4 \mathrm{~nm}$ Bi nanowires is due to localization effects. Therefore, there is an optimum wire diameter, on the order of $9 \mathrm{~nm}$ in Bi. Size-quantization effects enhance the Seebeck coefficient with decreasing wire diameter at diameters above the optimum, but localization effects decrease the Seebeck coefficient at diameters below the optimum. 


\subsection{Thermal conductivity}

Figure 11 shows the temperature dependence of the thermal conductance of some samples of $\mathrm{Bi} / \mathrm{Al}_{2} \mathrm{O}_{3}$ and $\mathrm{Zn} / \mathrm{Al}_{2} \mathrm{O}_{3}$ nanowire composites [40], along with the thermal conductivity of bulk $\mathrm{Bi}[26]$. Irrespective of the material in the pores, the conductance shows a decrease from room temperature to a plateau, followed by a $T^{n}$ law upon further cooling, with $n \approx 1.2$ to 1.4 . The thermal conductiv-

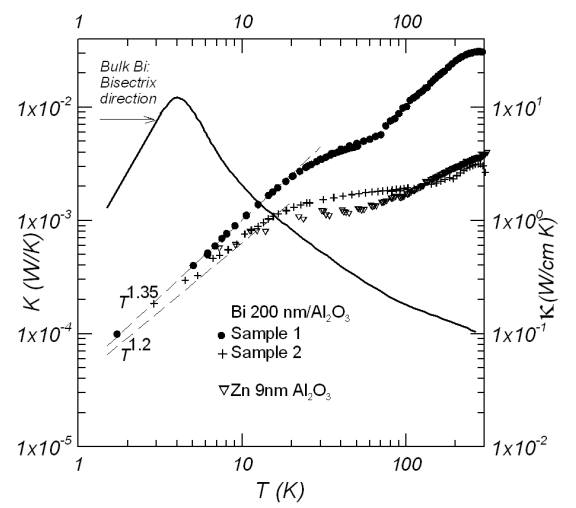

Fig. 11. Temperature dependence of the thermal conductivity of bulk Bi [26] (right ordinate) compared to the thermal conductance of 2 samples of $200 \mathrm{~nm}$ Bi nanowires imbedded in oriented anodic alumina, and of one sample of $9 \mathrm{~nm} \mathrm{Zn}$ nanowires in randomly oriented porous alumina (left ordinate axis) [40].

ity of bulk $\mathrm{Bi}$, which is phonon-dominated, follows the characteristic temperature dependence of the lattice thermal conductivity of dielectric crystals. From this, we conclude that the alumina matrix conducts most of the heat, even though the thermal conductivity of $\mathrm{Bi}$ is 2 orders of magnitude larger than that of glasses at room temperature. Still, it is worth pointing out one discrepancy: the observed temperature dependence is somewhat different of that of a glass, where the exponent $n \geq 1.8$. A power law exponent lower than the expected value suggests the following thoughts. Firstly, we would have expected the heat conduction of the $\mathrm{Bi}$ nanowires in that temperature range to be one-dimensional, as the dominant phonon wavelength at $10 \mathrm{~K}$ in bulk $\mathrm{Bi}$ is $160 \mathrm{~nm}$ [40], and this would lead to a thermal conductance with $n=1$. Secondly, for $\mathrm{Zn}$ nanowires with a resistance given in Fig. 11, the Wiedemann-Franz law would lead to an electronic thermal conductance with $n=1 / 2$. Nevertheless, while the observed temperature dependence raises questions, the magnitude of the thermal conductance is compatible with a thermal short through the porous host material. This makes it impossible for the Bi nanowire composites to achieve a high value of $Z T$ with the current state of the technology. Nevertheless, Bi nanowires are an excellent scientific platform on which the concepts of low-dimensional thermoelectric materials have been tested. 


\subsection{Other quantum-wire systems}

Several extensions to the work on bismuth nanowires have been reported, which we briefly review here.

Firstly, the work on Bi nanowires has been extended to the isoelectronic $\mathrm{Bi}_{1-x} \mathrm{Sb}_{x}$ alloys. Below about $150 \mathrm{~K}$, these alloys in bulk form are excellent thermoelectric materials [41] and are semiconductors for $7 \%<x<22 \%$. The situation above $150 \mathrm{~K}$ is less clear, as it is known [35] that the band structure of the semimetal $\mathrm{Bi}$ is quite temperature-dependent, but the mixed effects of Sb-alloying and temperature are unknown at this point. $\mathrm{Bi}_{1-x} \mathrm{Sb}_{x}$ alloy quantum wires are expected to have a better thermoelectric performance than Bi wires of the same diameter, especially for $p$-type material. It has been possible to calculate a phase diagram of $\mathrm{Bi}_{1-x} \mathrm{Sb}_{x}$ alloy nanowires as a function of $\mathrm{Sb}$ concentration and nanowire diameter [42]. The phase diagram predicts two semimetal regimes, one Bi-like with holes at the $T$-point and one Sb-like with holes at the $H$-point, and two indirect semiconducting regimes with gaps between $L$-point electrons and either $T$ or $H$-point holes. There is one singular point at the condition where the 10 hole pockets (about the $T$-point, the $3 L$-points and the $6 H$-points in the Brillouin zone) coalesce in energy. At this point (wire diameter of $60 \mathrm{~nm}$, and $x=13 \%$ ) the density of states is particularly high, which is beneficial for increasing the magnitude of the Seebeck coefficient. The diagram also predicts that the critical diameter at which the overlap between the $L$-point electron subbands and the $T$-point hole subbands vanishes (semimetal-to-semiconduc tor transition), is shifted to higher diameters as $\mathrm{Sb}$ is added to pure $\mathrm{Bi}$. We therefore expect to obtain better thermoelectric performance in $\mathrm{Bi}_{1-x} \mathrm{Sb}_{x}(x \approx 20 \%)$ when compared to pure Bi nanowires.

Secondly, the calculations have been extended to the case of segmented nanowires, in which alternate regions of two materials are grown along the length of the nanowire like segments on a bamboo stick. The thermoelectric properties of such quasi-zero-dimensional structures have been calculated for the case of $\mathrm{PbTe} / \mathrm{PbSe}$ and $\mathrm{PbSe} / \mathrm{PbS}$ segmented nanowires [43], and $Z T$ values considerably higher than in nanowires made from random alloys of comparable composition are expected.

\section{Quantum-dot systems}

\subsection{Quantum-dot superlattices}

Springholz et al. $[44,45]$ grew $\mathrm{PbSe} / \mathrm{PbEuTe}$ superlattices in which a three-dimensional array of islands formed spontaneously, with dimensions small enough that they can be considered as quantum dots. Harman and co-workers [10] developed MBE grown $\mathrm{PbTe} / \mathrm{PbTe}_{1-x} \mathrm{Se}_{x}$ quantum dot superlattices (QDSL), in which small quantum dots with a higher Se content grew at the interface between the $\mathrm{PbTe}$ regions and the alloy regions. Figure 12 shows [46] the structure of 

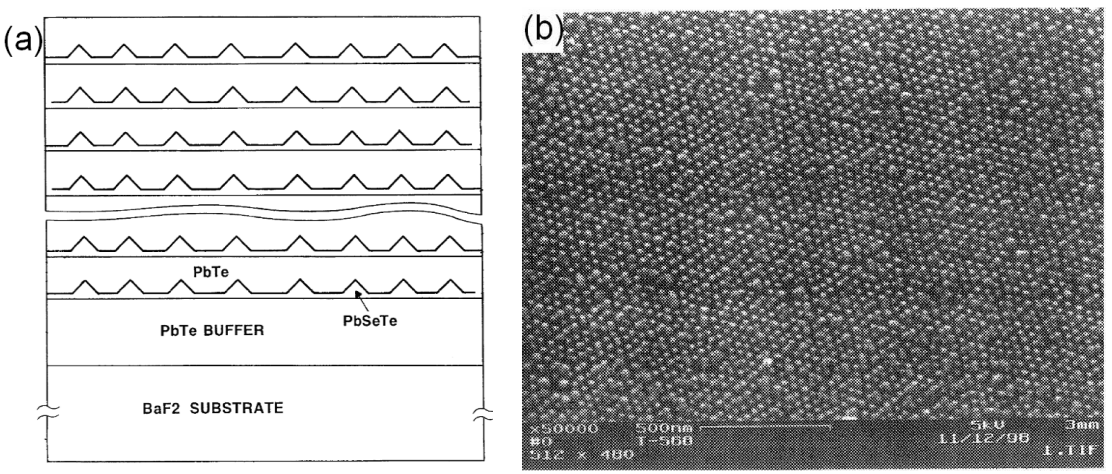

Fig. 12. Structure (a) and plan-view scanning electron micrograph (b) of a $\mathrm{PbTe} / \mathrm{PbSe}_{1-x} \mathrm{Te}_{x}$ quantum-dot superlattice (after [46]).

such superlattice and a scanning electron microscope image of it. When viewed from a 3D standpoint, these quantum dot superlattices can be interpreted as a $\mathrm{PbTe}$ host into which quantum dots are introduced in a periodic way in all three $(x, y, z)$ directions.

This technique creates materials with a high thermoelectric figure of merit: in 2000, Harman et al. reported [47] $Z T=0.9$ at $300 \mathrm{~K}$, double the $Z T$ of $\mathrm{PbTe}$, which in bulk material reaches a maximum of 0.45 . In 2002 they reported [10] $Z T=2$ at $300 \mathrm{~K}$ in $\mathrm{PbTe} / \mathrm{Pb}_{1-y} \mathrm{Sn}_{y} \mathrm{Te}_{1-x} \mathrm{Se}_{x}$ QDSLs with $x=98 \%$ and $y=16 \%$. Later yet [11], QDSLs were reported to have reached $Z T=3$ at $450 \mathrm{~K}$. What mechanisms are responsible for these results?

Firstly, the lattice thermal conductivity is strongly reduced. The thermal conductivity for the $n$-type PbSeTe/PbTe QDSLs is [10] on the order of 0.58 to $0.62 \mathrm{~W} /(\mathrm{m} \mathrm{K})$. Subtracting an estimate for electronic thermal conductivity $\kappa_{E}$ made using the Wiedemann-Franz law and the electrical conductivity, this leaves $\kappa_{\mathrm{L}} \approx 0.33 \mathrm{~W} /(\mathrm{m} \mathrm{K})$ (lower values yet are possible in quaternary $\mathrm{Pb}_{0.84} \mathrm{Sn}_{0.16} \mathrm{Se}_{0.98} \mathrm{Te}_{0.02} / \mathrm{PbTe}$ QDSLs). The lattice thermal conductivity of an equivalent $16 \%$ random $\mathrm{PbSeTe}$ alloy is [3] $1.25 \mathrm{~W} /(\mathrm{m} \mathrm{K})$, so that the first effect of the quantum dots is reduce $\kappa_{\mathrm{L}}$ by a factor of almost 4 , and probably more for the quaternary QDSL. On the other hand, the electron mobility of a similar PbSeTe alloy at a very low doping level is $[3] 1,000 \mathrm{~cm}^{2} /(\mathrm{V} \mathrm{s})$, and the $300 \mathrm{~K}$ mobility in lead salts is not [48] very sensitive to doping levels up to $2 \times 10^{19} \mathrm{~cm}^{-3}$. Compared to the ternary $n$-type QDSLs, the mobility reduction is only on the order of 3 or somewhat less.

The second mechanism increasing $Z T$ in QDSLs is a boost in the Seebeck coefficient over that of bulk material of the same carrier density. Figure 13 shows the room-temperature absolute value of the Seebeck coefficient of the samples described in Ref. [10] (crosses) as a function of the carrier density, alongside with data for a bulk sample (open dot) and other samples that will be described later (full dots). The full line gives the Seebeck coefficient for bulk PbTe, assuming 


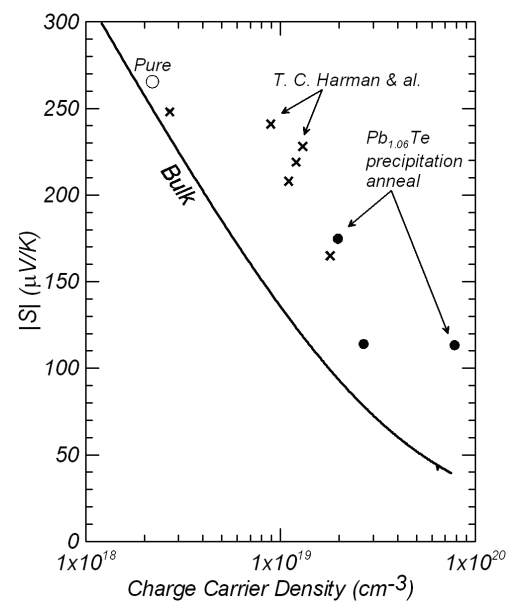

Fig. 13. The absolute value of the Seebeck coefficient of $\mathrm{PbTe}$ as a function of the carrier density. The full line gives the dependence for bulk $\mathrm{PbTe}$, assuming acoustic phonon scattering, and the open dot is an experimental data point. The crosses are the data of Harman et al., the full dots correspond to measurements taken on $\mathrm{Pb}$-rich $\mathrm{PbTe}$ with nanoprecipitates of metallic $\mathrm{Pb}$.

acoustic phonon scattering. This plot shows that the thermopower is considerably enhanced in the QDSLs, compared to bulk samples. As, for the case of $\mathrm{Pb}_{0.98} \mathrm{Sn}_{0.02} \mathrm{Se}_{0.13} \mathrm{Te}_{0.87}$ QDSLs the mobility remains above $500 \mathrm{~cm}^{2} /(\mathrm{V} \mathrm{s})$, the power factor $S^{2} \sigma$ is quite impressive. The exact mechanism for this enhancement is not clear at the moment, and several theories are emerging, based on either scattering (see Eq. (6)) or other [22, 23] energy filtering ideas, as outlined in the introduction. We shall see further, in the case of PbAgSbTe alloys, that there are more possibilities yet.

\subsection{Nanoprecipitates in bulk PbTe}

The MBE-grown films described above [10] provide the proof-of-principle that materials with $Z T=2$ do exist, but they can only be prepared in very small quantities and are thus suited mostly to applications that do not require large power dissipations. It would be desirable for widespread applications to synthesize similar materials in bulk form. Grinding and sintering nanograin thermoelectric materials offer one approach, used in both $\mathrm{PbTe}$ [49] and skutterudites [50], and, as in QDSLs, the thermal conductivity is reduced. Unfortunately, the mobility is reduced too, but this is partially offset by an increase in the Seebeck coefficient in the nanograined material compared to the Seebeck in bulk material of similar carrier density $[49,50]$. It was shown [49] that this is due to an increase in the energy dependence of the relaxation time, the "scattering parameter", and we will summarize that experimental proof here, but using a more favorable thermoelectric material system: PbTe with nanoprecipitates. 
It has been [51] known for a long time that bulk lead-rich $\mathrm{PbTe}$ samples can be prepared with lead inclusions. Using conventional metallurgical heat treatments analogous to those developed for the precipitation hardening of aluminum alloys [52, 53], bulk $\mathrm{PbTe}$ sample enriched with $6 \% \mathrm{~Pb}$ were prepared and analyzed recently [54] that contained nanometer-scale precipitates of metallic $\mathrm{Pb}$. The evidence the authors present for the presence of metallic $\mathrm{Pb}$ is threefold: $\mathrm{X}$-ray diffraction (XRD), scanning electron micrography (SEM), and a superconductive phase transition. A conventional Scherer analysis [55] was then used to determine the crystallite size of the $\mathrm{Pb}$ precipitates, which were on the order of 30 to $40 \mathrm{~nm}$. When left undoped, the $\mathrm{Pb}$-rich $\mathrm{PbTe}$ samples are $n$-type, but they can be counter-doped $p$-type with thallium. The authors [54] also report the behavior of Ag-doped PbTe. At low concentrations $\left(<\right.$ a few $\left.10^{19} \mathrm{~cm}^{-3}\right)$ silver is a conventional $p$-type dopant [56]. PbTe doped with Ag in the high- $10^{19} \mathrm{~cm}^{-3}$ range turns out $n$-type, and displays XRD spectra, SEM images, and a partial superconductive transition similar to the Pb-rich samples. This suggests the presence of metallic $\mathrm{Pb}$ inclusions, perhaps because $\mathrm{Ag}$ binds preferentially with $\mathrm{Te}$, liberating $\mathrm{Pb}$ atoms to precipitate. An SEM image of such $\mathrm{PbTe} \mathrm{Ag}$ sample is shown in Fig. 14.

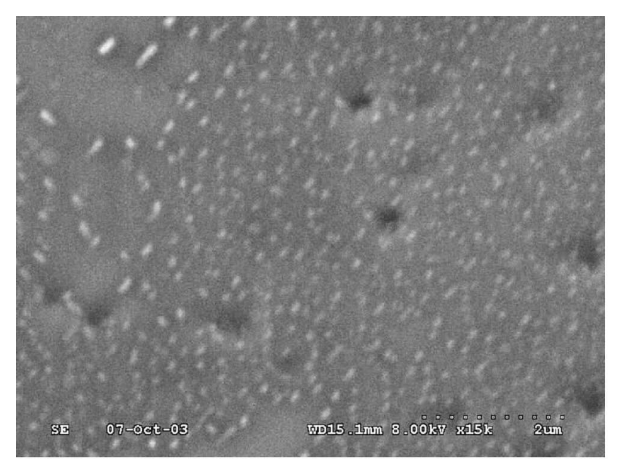

Fig. 14. Scanning electron micrographs of a $\mathrm{PbTe}: \mathrm{Ag}$ sample showing nanoprecipitates (after [54]).

The transport properties of PbTe samples with nanoprecipitates are summarized as follows. Firstly, the lattice thermal conductivity of all samples in this study is calculated to be on the order of $2.5 \mathrm{~W} /(\mathrm{m} \mathrm{K})$ at $300 \mathrm{~K}$. Secondly, we show the absolute value of the Seebeck coefficient of both $n$ and $p$-type doped PbTe with $6 \%$ excess $\mathrm{Pb}$ as a function of the carrier density at $300 \mathrm{~K}$ in Fig. 13, alongside the bulk data and those on QDSLs [10]. The Seebeck coefficient is enhanced almost as much as for the QDSLs, and this was observed on the heavily-doped PbTe:Ag samples as well, but not on Pb-rich PbTe with only $3 \% \mathrm{~Pb}$. Thirdly, the power factor $S^{2} \sigma$ reaches $20 \mu \mathrm{W} /\left(\mathrm{cm} \mathrm{K}^{2}\right)$, on par with optimally doped PbTe [2], due once again to the effect of the nanoprecipitates on the mobility. The mobility of 
$p$-type $\mathrm{PbTe}: \mathrm{Tl}$ sample is reduced dramatically, while that of $n$-type $\mathrm{Pb}$-rich $\mathrm{PbTe}$ is reduced by a factor of 3.5 compared to similarly prepared bulk PbTe.

In order to analyze the physical origin of the increase in the Seebeck coefficient, the authors applied the "method of the four coefficients" [49, 57]. In essence, at each temperature, there are four unknown quantities for a given sample: the carrier density, the carrier mobility, the effective mass, which can be enhanced by size-quantization effects or other mechanisms that affect the density of states, and the energy dependence of the relaxation time, which we choose to represent by the scattering exponent $\lambda$ in Eq. (6). In order to solve this system uniquely without "fitting" some parameters, we need four experimental data, and these we choose to be the resistivity, the Seebeck coefficient, the Hall coefficient, and the transverse Nernst-Ettingshausen coefficient. The thermoelectric power $S$, the carrier concentration $p$, and the electrical conductivity $\sigma$ are functions of the Fermi energy $E_{\mathrm{F}}$ in the system, the carrier effective mass and the carrier scattering relaxation time $\tau$, which is given (Eq. (6)) for simple parabolic bands. The assumption that the bands are parabolic does not hold for the valence band of PbTe, for which [56] the Fermi surfaces are ellipsoids of revolution, and the energy dispersion relation is

$$
\frac{\hbar^{2} k_{\mathrm{l}}^{2}}{2 m_{\mathrm{l}}{ }^{*}}+\frac{\hbar^{2} k_{\mathrm{t}}^{2}}{m_{\mathrm{t}}{ }^{*}}=\gamma(E)=E\left(1+\frac{E}{E_{\mathrm{g}}}\right),
$$

where $E_{\mathrm{g}}$ is the direct energy gap of $\mathrm{PbTe}$, and $k$ and $m^{*}$ are the electron wave vector and effective mass (at $k=0$ ) along the longitudinal (suffix l) and transverse (suffix t) directions of the ellipsoids. The density of states is [56]:

$$
g(E)=\frac{\sqrt{2}\left(m_{\mathrm{d}}{ }^{*}\right)^{3 / 2}}{\pi^{2} \hbar^{3}} \gamma^{\prime} \sqrt{\gamma(E)},
$$

where $\gamma^{\prime}$ is the derivative of $\gamma$ with respect to $E$, $\hbar$ is the Planck constant, and $m_{\mathrm{d}}{ }^{*}=\left(m_{\mathrm{l}} m_{\mathrm{t}}^{2}\right)^{1 / 3}$ is the density of states effective mass in which we include the degeneracy number when the Fermi surface contains more than one pocket. We saw that Eq. (6) contained two factors, the density of states and the transition matrix element; for non-parabolic bands it follows that the energy dependence of $\tau_{\mathrm{e}}$ is given by $[56]$ :

$$
\tau_{\mathrm{e}}=\tau_{0} \frac{\gamma(E)^{\lambda-1 / 2}}{\gamma^{\prime}(E)} .
$$

The carrier density is now

$$
p=\frac{\left(2 m_{\mathrm{d}}^{*} k_{\mathrm{B}} T\right)^{3 / 2}}{3 \pi^{2} \hbar^{3}} \int_{0}^{\infty} \gamma(z)^{3 / 2}\left(-\frac{\partial f_{0}}{\partial z}\right) \mathrm{d} z,
$$

where $z=E / k_{\mathrm{B}} T$ is the reduced energy, so that $\gamma(z)$ is given by (11) but with $z$ substituting for $E$. $f_{0}$ is the Fermi-Dirac distribution function. The electrical conductivity is [56]: 


$$
\sigma=\frac{\left(2 m_{\mathrm{d}}{ }^{*} k_{\mathrm{B}} T\right)^{3 / 2}}{3 \pi^{2} \hbar^{3}} \frac{e^{2}}{m_{\alpha}^{*}} \int_{0}^{\infty} \frac{\gamma(z)^{3 / 2}}{\gamma^{\prime}(z)} \tau(z)\left(-\frac{\partial f_{0}}{\partial z}\right) \mathrm{d} z,
$$

where $e$ is the carrier charge, $m_{\alpha}^{*}$ is the effective mass along the crystallographic direction of the sample studied, or the appropriate average mass for polycrystals, and $\gamma^{\prime}(z)$ is the derivative of $\gamma(z)$ with respect to $z$. The low-field $(\mu B \ll 1)$ Hall coefficient is $[56]$ :

$$
\begin{aligned}
R_{H} & =\frac{3 K(K+2)}{(2 K+1)^{2}} \frac{1}{p e} \\
& \times \frac{\left[\int_{0}^{\infty} \frac{\gamma(z)^{3 / 2}}{\left(\gamma^{\prime}(z)\right)^{2}} \tau^{2}(z)\left(-\frac{\partial f_{0}}{\partial z}\right) \mathrm{d} z\right]\left[\int_{0}^{\infty} \gamma(z)^{3 / 2}\left(-\frac{\partial f_{0}}{\partial z}\right) \mathrm{d} z\right]}{\left[\int_{0}^{\infty} \frac{\gamma(z)^{3 / 2}}{\gamma^{\prime}(z)} \tau(z)\left(-\frac{\partial f_{0}}{\partial z}\right) \mathrm{d} z\right]^{2}},
\end{aligned}
$$

where $K$ is the effective mass anisotropy coefficient, $K=m_{\mathrm{l}} / m_{\mathrm{t}}$. The Seebeck coefficient is [56]:

$$
S=\frac{k_{\mathrm{B}}}{e}\left[\frac{\int_{0}^{\infty} \frac{\gamma(z)^{3 / 2}}{\gamma^{\prime}(z)} z \tau(z)\left(-\frac{\partial f_{0}}{\partial z}\right) \mathrm{d} z}{\int_{0}^{\infty} \frac{\gamma(z)^{3 / 2}}{\gamma^{\prime}(z)} \tau(z)\left(-\frac{\partial f_{0}}{\partial z}\right) \mathrm{d} z}-\frac{E_{\mathrm{F}}}{k_{\mathrm{B}} T}\right]
$$

and the low-field $(\mu B \ll 1)$ isothermal Nernst coefficient is [56]:

$$
\begin{aligned}
N= & R_{H} \sigma \frac{k_{\mathrm{B}}}{e} \\
& \times\left[\frac{\int_{0}^{\infty} \frac{\gamma(z)^{3 / 2}}{\left(\gamma^{\prime}(z)\right)^{2}} z \tau^{2}(z)\left(-\frac{\partial f_{0}}{\partial z}\right) \mathrm{d} z}{\int_{0}^{\infty} \frac{\gamma(z)^{3 / 2}}{\left(\gamma^{\prime}(z)\right)^{2}} \tau^{2}(z)\left(-\frac{\partial f_{0}}{\partial z}\right) \mathrm{d} z}-\frac{\int_{0}^{\infty} \frac{\gamma(z)^{3 / 2}}{\gamma^{\prime}(z)} z \tau(z)\left(-\frac{\partial f_{0}}{\partial z}\right) \mathrm{d} z}{\int_{0}^{\infty} \frac{\gamma(z)^{3 / 2}}{\gamma^{\prime}(z)} \tau(z)\left(-\frac{\partial f_{0}}{\partial z}\right) \mathrm{d} z}\right] .
\end{aligned}
$$

Solving now Eqs. (13)-(18) at each temperature with the experimental values [54] measured for the resistivity $\rho$, the thermoelectric power $S$, the low-field Hall coefficient $R_{H}$, and low-field isothermal* Nernst coefficient $N$, one can deduct the density-of-states effective mass and the scattering parameter. The authors observe first that in all cases, the resulting value for the density-of-states effective mass remains at $m_{\mathrm{d}}^{*}=0.12 \pm 0.04$ free electron masses, around the value reported [58] for PbTe. Values for the scattering parameter $\lambda$ are reported in Fig. 15: for the bulk sample $\lambda=0.2 \pm 0.2$, indicative of the dominance of acoustic phonon scattering. When applied to the samples with $\mathrm{Pb}$ precipitates the procedure gives values of $\lambda$ between 2.5 and 4, as shown in Fig. 15, irrespective of the carrier type. We therefore conclude that an enhancement in the energy dependence of

${ }^{*}$ Experimentally one measures the transverse Nernst-Ettingshausen coefficient by applying a temperature gradient to the sample and measuring a transverse voltage; the sample is thus mounted adiabatically, and the quantity measured is the adiabatic Nernst coefficient, not the isothermal one. The reader is referred to the original publications $[49,54]$ for the correction factors to convert one to the other: in thermoelectric materials, like in all heavily-doped narrow-gap semiconductors, these corrections can be substantial. 


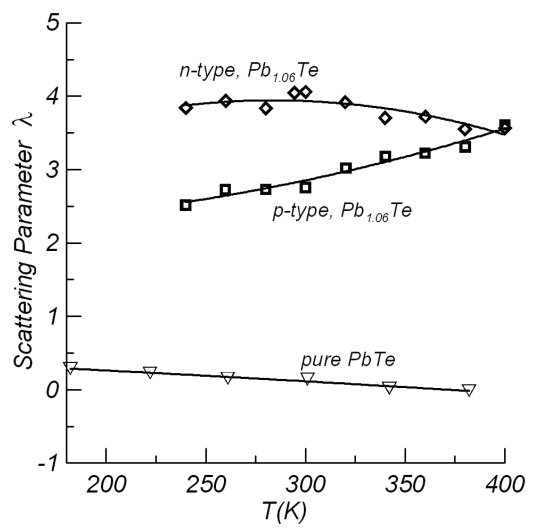

Fig. 15. Temperature dependence of the scattering parameter $\lambda$ of bulk $\mathrm{PbTe}$ with and without $(\nabla)$ nanoinclusions (after [54]).

the scattering time is at the origin of the increase in the Seebeck coefficient in samples that include $\mathrm{Pb}$ nanoprecipitates.

$$
\text { 3.3. } \mathrm{AgPb}_{m} \mathrm{SbTe}_{2+m}
$$

Another recent development in the field of bulk materials with nanometer-scaled inclusions, we mention the recently reported $\mathrm{AgPb}_{m} \mathrm{SbTe}_{2+m}$ alloys [59] that have $Z T=2.1$ at $800 \mathrm{~K}$. The role of the nanoinclusions in $\mathrm{AgPb}_{m} \mathrm{SbTe}_{2+m}$ is discussed in a subsequent paper [60]. Metallurgically, these bulk alloys are [61] solutions of PbTe and $\mathrm{AgSbTe}_{2}$, the PbTe-equivalent of the GeTe-based TeAgGeSb (TAGS) alloys [62], which also have nanometer inclusions [63]. This simple view is not quite correct, however, and Bilc et al. point out that the material should be regarded [60] as a PbTe lattice with $\mathrm{Ag}$ and $\mathrm{Sb}$ inclusions. $\mathrm{Ag}$ atoms introduce new states near the top of the valence band of $\mathrm{PbTe}$; isolated $\mathrm{Sb}$ atoms introduce resonant states near the bottom of the $\mathrm{PbTe}$ conduction band. The $\mathrm{Ag}-\mathrm{Sb}$ pairs result in an increase in the density of states, compared to that of pure $\mathrm{PbTe}$, right around the band gap. As a result of the Mott equation once again, the Seebeck coefficient, and thus the power factor $S^{2} \sigma$ are increased. The ubiquitous first mechanism that boosts $Z T$ in nanostructures, the reduction of the lattice thermal conductivity, is also at work.

\section{Summary}

Thermoelectric technology has been one of the beneficiaries of the recent progress in nanotechnologies as applied to semiconductor structures. Quantum-dot superlattices have experimentally shown [10] an improvement in thermoelectric figure of merit by a factor of at least two over the best bulk thermoelectric materials. In this review, we have summarized the physical origins of this improvement. 
The optimization of a thermoelectric material involves three parameters, the electrical and thermal conductivities $(\sigma$ and $\kappa)$, and the Seebeck coefficient $S$, which are inter-dependent and have to be optimized as a whole. The use of low-dimensional structures adds a new variable, which relaxes this inter-dependence, and allows us to find a more favorable optimum. There are two main mechanisms through which nanometer-scale materials improve the figure of merit, a reduction of the lattice thermal conductivity, and an enhancement of the product $S^{2} \sigma$. The latter effect has been shown experimentally to arise through several physical mechanisms: size-quantization effects in quantum wires (Bi), enhancement of the local density of states in solids in which there are nanometer-sized inclusions $\left(\mathrm{AgPb}_{m} \mathrm{SbTe}_{2+m}\right)$, and electron energy filtering on nano-precipitates $(\mathrm{PbTe}+6 \% \mathrm{~Pb}$ metal). The review also cites theoretical papers that outline more possibilities.

\section{Acknowledgments}

The author acknowledges useful discussions with Dr. D.T. Morelli and Professor M.S. Dresselhaus. He also thanks Professors T. Story and G. Karczewski for critical reading of the manuscript.

\section{References}

[1] M. Telkes, Int. J. Appl. Phys. 18, 1116 (1947).

[2] A.F. Ioffe, Semiconductor Thermoelements and Thermoelectric Cooling, Inforsearch Ltd., London 1957.

[3] A.F. Ioffe, Physics of Semiconductor, Academic Press Inc., New York 1960.

[4] H.J. Goldsmid, Applications of Thermoelectricity, Methuen, London 1960.

[5] G.S. Nolas, J. Sharp, H.J. Goldsmid, Thermoelectricity, Springer-Verlag, Berlin 2000 .

[6] J.-P. Fleurial, A. Borshchevsky, T. Caillat, D.T. Morelli, G.P. Meisner, in: Proc. 15th Int. Conf. on Thermoelectrics, Eds. T. Caillat, J.-P. Fleurial, A. Borshchevsky, IEEE catalog number 96TH8169, Piscataway (NJ) 1996, p. 91; D.T. Morelli, T. Caillat, J.-P. Fleurial, A. Borshchevsky, J. Vandersande, B. Chen, C. Uher, Phys. Rev. B 51, 9622 (1995).

[7] L.D. Hicks, M.S. Dresselhaus, Phys. Rev. B 47, 12727-31 (1993).

[8] L.D. Hicks, M.S. Dresselhaus, Phys. Rev. B 47, 16631-4 (1993).

[9] R. Venkatasubramanian, E. Siivola, T. Colpitts, B. O'Quinn, Nature 413, 597 (2001).

[10] T.C. Harman, P.J. Taylor, M.P. Walsh, B.E. LaForge, Science 297, 2229-32 (2002).

[11] T.C. Harman, unpublished.

[12] J.P. Heremans, Mater. Res. Soc. Symp. Proc. 793, 3-14 (2004).

[13] J.W. Fenton, J.S. Lee, R.J. Buist, United States Patent 4,065,936, Jan. 3, 1978. 
[14] L. Bell, in: Proc. 21st Int. Conf. on Thermoelectrics, ICT'02, Long Beach, California (USA) 2002, Eds. T. Caillat, J. Snyder, IEEE, Piscataway (NJ) 2002, p. 447.

[15] P.E. Gray, The Dynamic Behavior of Thermoelectric Devices, Wiley, New York 1961.

[16] M. Cutler, N.F. Mott, Phys. Rev. 181, 1336 (1969).

[17] Equation (8.63) in N.W. Ashcroft, N.D. Mermin, Solid State Physics, Holt Rinehart and Winston, Philadelphia 1976.

[18] A.Ya. Shik, Fiz. Tekh. Poluprovodn. 7, 261 (1973) [Sov. Phys.-Semiconductors 7, 1 87-92 (1973)].

[19] W.F. Leonard, T.J. Martin, Jr., Electronic Structure and Transport Properties of Crystals, Krieger Publ. Co., Malabar, FL, 1979.

[20] T.S. Stavitskaya, L.S. Stilbans, Sov. Phys. Solid State 2, 1868 (1961).

[21] J.-P. Issi, J.-P. Michenaud, J. Heremans, Phys. Rev. B 14, 5156 (1976).

[22] T.E. Humphrey, H. Linke, Phys. Rev. Lett. 94, 096601 (2005).

[23] A. Shakouri, J.D. Bowers, Appl. Phys. Lett. 71, 1234 (1997).

[24] J. Tauc, Photo and Thermoelectric Effects in Semiconductors, Pergamon Press, New York 1962.

[25] Y.-M. Lin, X. Sun, M.S. Dresselhaus, Phys. Rev. B 62, 4610 (2000).

[26] C.F. Gallo, B.S. Chandrasekhar, P.H. Sutter, J. Appl. Phys. 34, 144 (1963).

[27] Z. Zhang, X. Sun, M.S. Dresselhaus, J.Y. Ying, J. Heremans, Phys. Rev. B 61, 4850 (2000).

[28] D.A. Glocker, M.J. Skove, Phys. Rev. B 15, 608 (1977).

[29] J.P. Heremans, C.M. Thrush, Y.-M. Lin, S.B. Cronin, Z. Zhang, M.S. Dresselhaus, J.F. Mansfield, Phys. Rev. B 61, 2921 (2000).

[30] C.M. Thrush, J.P. Heremans, United States Patent Number 6,159,831 (2000).

[31] J.P. Heremans, C.M. Thrush, D.T. Morelli, M.C. Wu, Phys. Rev. Lett. 88, 216801 (2002).

[32] J.-P. Michenaud, J.-P. Issi, J. Phys. C, Solid State Phys. 5, 3061 (1972).

[33] J.P. Heremans, in: Proc. 22nd Int. Conf. on Thermoelectrics, La Grande Motte (France) 2003, Eds. H. Scherrer, J.-C. Tedenac, IEEE, Piscataway (NJ) 2003, p. 324.

[34] J.P. Heremans, C.M. Thrush, Phys. Rev. B 59, 12579 (1999).

[35] J. Heremans, O.P. Hansen, J. Phys. C, Solid State Phys. 12, 3483 (1979).

[36] D. Beutler, J. Giordano, Phys. Rev. B 38, 8 (1988).

[37] J. Heremans, C.M. Thrush, Z. Zhang, X. Sun, M.S. Dresselhaus, J.Y. Ying, D.T. Morelli, Phys. Rev. B 58, R10091 (1998).

[38] J. Heremans, C.M. Thrush, Y.-M. Lin, S.B. Cronin, M.S. Dresselhaus, Phys. Rev. $B$ 63, 085406 (2001).

[39] J.P. Heremans, C.M. Thrush, D.T. Morelli, M.C. Wu, Phys. Rev. Lett. 91, 076804 (2003).

[40] J.P. Heremans, in: Thermal Conductivity 25, Eds. D.T. Morelli, C. Uher, CRC Pr Llc, Lancaster, PA 1999, p. 114. 
[41] B. Lenoir, M. Cassart, J.-P. Michenaud, H. Scherrer, S. Scherrer, J. Phys. Chem. Solids 57, 89 (1996).

[42] O. Rabin, Yu-Ming Lin, M.S. Dresselhaus, Appl. Phys. Lett. 79, 81 (2001).

[43] Yu-Ming Lin, M.S. Dresselhaus, Phys. Rev. B 68, 075304S (2003).

[44] G. Springholz, V. Holy, M. Pinczolits, G. Bauer, Science 282, 734 (1998).

[45] G. Springholz, M. Pinczolits, P. Mayer, V. Holy, G. Bauer, H.H. Kang, L. Salamanca-Riba, Phys. Rev. Lett. 84, 4669 (2000).

[46] T.C. Harman, P.J. Taylor, M.P. Walsh, United States Patent Number 6,605,772 B2 (2003).

[47] T.C. Harman, P.J. Taylor, D.L. Spears, M.P. Walsh, J. Electron. Mater. 29, L1 (2000).

[48] Yu.I. Ravich, B.A. Efimova, V.I. Tamarchenko, Phys. Status Solidi B 43, 453 (1971).

[49] J.P. Heremans, C.M. Thrush, D.T. Morelli, Phys. Rev. B 70, 115334 (2004).

[50] X. Shi, L. Chen, J. Yang, G.P. Meisner, Appl. Phys. Lett. 84, 2301 (2004).

[51] A. Lasbley, R. Granger, S. Rolland, Solid State Commun. 13, 1045 (1973).

[52] A. Guinier, Nature 142, 569 (1938).

[53] G.D. Preston, Nature 142, 570 (1938).

[54] J.P. Heremans, C.M. Thrush, D.T. Morelli, J. Appl. Phys., submitted for publication.

[55] B.D. Cullity, Elements of X-Ray Diffraction, Addison-Wesley, Reading, MA 1956.

[56] Yu. Ravich, B.A. Efimova, I.A. Smirnov, Semiconducting Lead Chalcogenides, Plenum Press, New York 1970.

[57] A. Chernik, V.I. Kaidanov, M.I. Vinogradova, N.V. Kolomoets, Sov. Phys. Semicond. 2, 645 (1968).

[58] H. Preier, Appl. Phys. 20, 189 (1979).

[59] K.F. Hsu, S. Loo, F. Guo, W. Chen, J.S. Dyck, C. Uher, T. Hogan, E.K. Polychroniadis, M.G. Kanatzidis, Science 303, 818 (2004).

[60] D. Bilc, S.D. Mahanti, E. Quarez, K.-F. Hsu, R. Pcionek, M.G. Kanatzidis, Phys. Rev. Lett. 93, 146403 (2004); Bull. Am. Phys. Soc. 50, 1407 (2005).

[61] T. Irie, T. Takahama, T. Ono, Jap. J. Appl. Phys. 2, 72 (1963).

[62] F.D. Rosi, J.P. Dismukes, E.F. Hockings, Electrical Eng., 450 (June 1960).

[63] R.W. Armstrong, J.W. Faust, W.A. Tiller, J. Appl. Phys. 31, 1954 (1960). 